\section{APEM}

Advances in Production Engineering \& Management

Volume 9 | Number 4 | December 2014 | pp 159-167

http://dx.doi.org/10.14743/apem2014.4.184
ISSN 1854-6250

Journal home: apem-journal.org

Original scientific paper

\title{
Laser cladding of Ti-6Al-4V alloy with vanadium carbide particles
}

\author{
El-Labban, H.F. ${ }^{a}$, Mahmoud, E.R.I. ${ }^{a}{ }^{*}$, Al-Wadai, H. ${ }^{a}$ \\ ${ }^{a}$ Faculty of Engineering, King Khalid University, Abha, Saudi Arabia
}

\begin{abstract}
A B S T R A C T
The tribological properties of Ti-6Al-4V alloy are generally poor. This study was an attempt to produce a hardened surface layer on this alloy for longer service life during severe wear conditions. For this purpose, laser surface cladding of this alloy with vanadium carbide (VC) powder was performed using a YAG Fiber laser at power strengths of $1000 \mathrm{~W}, 1500 \mathrm{~W}$, and $2000 \mathrm{~W}$ and a travelling speed of $4 \mathrm{~mm} / \mathrm{s}$. Surface cladded layers of Ti-6Al-4V alloy metal matrix composite reinforced with VC particles were produced on the substrate under all processing conditions. The size of the cladding layer was increased by increasing the processing power. The cladding layer was well bonded to the substrate, especially at higher processing powers. The VC particles were homogenously distributed within the cladding layer at processing powers of $2000 \mathrm{~W}$ and $1500 \mathrm{~W}$, whilst it showed some clusters at a power of $1000 \mathrm{~W}$. Some of the VC particles were melted and re-solidified as fine long dendritic structures during the laser treatment. The cladding layer produced under all processing conditions exhibits remarkable improvement of hardness and wear resistance (almost twice). As the processing powers decreased, the surface of the cladding layers showed higher hardness. The cladding layer also showed improved corrosion resistance.
\end{abstract}

\section{ARTICLE INFO}

Keywords:

Laser cladding

Ti-6Al-4V alloy

VC powder

Surface microhardness

Wear and corrosion resistance

*Corresponding author: emahoud@kku.edu.sa (Mahmoud, E.R.I.)

Article history:

Received 2 August 2014

Revised 29 October 2014

Accepted 10 November 2014 


\section{References}

[1] Ochonogor, O.F., Meacock, C., Abdulwahab, M., Pityana, S., Popoola, A.P.I. (2012). Effects of Ti and TiC ceramic powder on laser-cladded Ti-6Al-4V in situ intermetallic composite, Applied Surface Science, Vol. 263, 591-596, doi: 10.1016/i.apsusc.2012.09.114.

[2] Lin, Y.-C., Lin, Y.-C. (2011). Microstructure and tribological performance of Ti-6Al-4V cladding with SiC powder, Surface and Coatings Technology, Vol. 205, No. 23-24, 5400-5405, doi: 10.1016/j.surfcoat.2011.06.001.

[3] Mahamood, R.M., Akinlabi, E.T., Shukla, M., Pityana, S. (2013). Scanning velocity influence on microstructure, microhardness and wear resistance performance of laser deposited Ti6Al4V/TiC composite, Materials and Design, Vol. 50, 656-666, doi: 10.1016/j.matdes.2013.03.049.

[4] Tian, Y.S., Chen, C.Z., Li, S.T., Huo, Q.H. (2005). Research progress on laser surface modification of titanium alloys, Applied Surface Science, Vol. 242, No. 1-2, 177-184, doi: 10.1016/i.apsusc.2004.08.011.

[5] Alabeedi, K.F., Abboud, J.H., Benyounis, K.Y. (2009). Microstructure and erosion resistance enhancement of nodular cast iron by laser melting, Wear, Vol. 266, No. 9-10, 925-933, doi: 10.1016/j.wear.2008.12.015.

[6] Heydarzadeh Sohi, M., Ebrahimi, M., Ghasemi, H.M., Shahripour, A. (2012). Microstructural study of surface melted and chromium surface alloyed ductile iron, Applied Surface Science, Vol. 258, No. 19, 7348-7353, doi: 10.1016/i.apsusc.2012.04.014.

[7] Benyounis, K.Y., Fakron, O.M.A., Abboud, J.H., Olabi, A.G., Hashmi, M.J.S. (2005). Surface melting of nodular cast iron by Nd-YAG laser and TIG, Journal of Materials Processing Technology, Vol. 170, No. 1-2, 127-132, doi: 10.1016/i.jmatprotec.2005.04.108.

[8] Vamsi Krishna, B., Misra, V.N., Mukherjee, P.S., Sharma, P. (2002). Microstructure and properties of flame sprayed tungsten carbide coatings, International Journal of Refractory Metals and Hard Materials, Vol. 20, No. 5-6, 355-374, doi: 10.1016/S0263-4368(02)00073-2.

[9] Hua, G., Huang, Y., Zhao, J., Wang, L., Tian, Z., Zhang, J., Zhang, Y. (2004). Plasma-sprayed ceramic coating by laser cladding of $\mathrm{Al}_{2} \mathrm{O}_{3}$ nano-particles, The Chinese Journal of Nonferrous Metals, Vol. 14, No. 2, 199-203.

[10] Zenker, R., Buchwalder, A., Rüthrich, K., Griesbach, W., Nagel, K. (2013). First results of a new duplex surface treatment for cast iron: electron beam remelting and plasma nitriding, Surface and Coatings Technology, Vol. 236, 58-62, doi: 10.1016/j.surfcoat.2013.06.118.

[11] Zhang, W.P., Liu, S. (2005). Microstructure of Fe-Ti-B composite coating prepared by laser cladding, The Chinese Journal of Nonferrous Metals, Vol. 15, No. 4, 558-564.

[12] Tian, Y.S., Chen, C.Z., Wang, D.Y., Wang, Z.L. (2004). Study on microstructures and mechanical properties of insitu formed multiphase coatings produced by laser cladding of titanium alloy with silicon and graphite powders, Chineese Journal of Lasers, Vol. 31, No. 7, 879-882.

[13] Chehrghani, A., Torkamany, M.J., Hamedi, M.J. Sabbaghzadeh, J. (2012). Numerical modeling and experimental investigation of TiC formation on titanium surface pre-coated by graphite under pulsed laser irradiation, Applied Surface Science, Vol. 258, No. 6, 2068-2076, doi: 10.1016/j.apsusc.2011.04.064.

[14] Yasavol, N., Abdollah-zadeh, A., Ganjali, M., Alidokht, S.A. (2013). Microstructure and mechanical behavior of pulsed laser surface melted AISI D2 cold work tool steel, Applied Surface Science, Vol. 265, 653-662, doi: 10.1016/j.apsusc.2012.11.070.

[15] Yilbas, B.S., Patel, F., Karatas, C. (2013). Laser controlled melting of HSLA steel surface with presence of $\mathrm{B}_{4} \mathrm{C}$ particles, Applied Surface Science, Vol. 282, 601-606, doi: 10.1016/j.apsusc.2013.06.018.

[16] Gadag, S.P., Srinivasan, M.N., Mordike, B.L. (1995). Effect of laser processing parameters on the structure of ductile iron, Materials Science and Engineering: A, Vol. 196, No. 1-2, 145-154, doi: 10.1016/0921-5093(94)09719-4.

[17] Vilar, R. (2014). 10.07 - Laser powder deposition, In: Hashmi, S. (ed.), Comprehensive Materials Processing, Vol. 10, Elsevier, 163-216, doi: 10.1016/ B978-0-08-096532-1.01005-0.

[18] Uematsu, Y., Kakiuchi, T., Tokaji, K., Nishigaki, K., Ogasawara, M. (2013). Effects of shot peening on fatigue behavior in high speed steel and cast iron with spheroidal vanadium carbides dispersed within martensitic-matrix microstructure, Materials Science and Engineering: A, Vol. 561, 386-93, doi: 10.1016/j.msea.2012.10.045.

[19] Pierson, H.O. (1996). Handbook of refractory carbides and nitrides: properties, characteristics, processing and applications, New Jersey, Noyes publications, USA.

[20] Li, Y., Li, G., Yang, D., Li, G. (2012). Microstructure evolution and mechanical properties in VC/SiC nanomultilayers, Applied Surface Science, Vol. 258, No. 24, 9856-9858, doi: 10.1016/i.apsusc.2012.06.042.

[21] Tjong, S.C., Haydn, C. (2004). Nanocrystalline materials and coatings, Materials Science and Engineering: R: Reports, Vol. 45, No. 1-2, 1-88, doi: 10.1016/j.mser.2004.07.001.

[22] Lugscheider, E., Knotek, O., Bobzin, K., Bärwulf, S. (2000). Tribological properties, phase generation and high temperature phase stability of tungsten- and vanadium-oxides deposited by reactive MSIP-PVD process for innovative lubrication applications, Surface and Coatings Technology, Vol. 133-134, 362-368, 10.1016/S02578972(00)00963-4.

[23] Frykholm, R., Andrén, H.-O. (2001). Development of the microstructure during gradient sintering of a cemented carbide, Materials Chemistry and Physics, Vol. 67, No. 1-3, 203-208, doi: 10.1016/S0254-0584(00)00440-5.

[24] Ye, F., Hojamberdiev, M., Xu, Y., Zhong, L., Zhao, N., Li, Y., Huang, X. (2013). Microstructure, microhardness and wear resistance of $\mathrm{VC}_{\mathrm{p}} / \mathrm{Fe}$ surface composites fabricated in situ, Applied Surface Science, Vol. 280, 297-303, doi: 10.1016/j.apsusc.2013.04.152.

[25] Hashe, N.G., Neethling, J.H., Berndt, P.R., Andrén, H.-O., Norgren, S. (2007). A comparison of the microstructures of WC-VC-TiC-Co and WC-VC-Co cemented carbides, International Journal of Refractory Metals and Hard Materials, Vol. 25, No. 3, 207-21, doi: 10.1016/j.ijrmhm.2006.05.001.

[26] Kąc, S., Kusiński, J. (2003). SEM and TEM microstructural investigation of high-speed tool steel after laser melting, Materials Chemistry and Physics, Vol. 81, No. 2-3, 510-512, doi: 10.1016/S0254-0584(03)00062-2. 


\title{
Lasersko nanašanje vanadijevega karbidnega prahu na zlitino Ti-6Al-4V
}

\author{
El-Labban, H.F. ${ }^{a}$, Mahmoud, E.R.I. ${ }^{\mathrm{a},{ }^{*},}$, Al-Wadai, H. ${ }^{\mathrm{a}}$ \\ ${ }^{a}$ Faculty of Engineering, King Khalid University, Abha, Saudi Arabia
}

\section{POVZETEK}

Tribološke lastnosti zlitine Ti-6Al-4V so razmeroma slabe. $\mathrm{V}$ raziskavi predlagamo obdelovalni postopek za utrditev površine omenjene zlitine, ki omogoča daljšo uporabo izdelkov pod zahtevnimi pogoji obrabe. Za ta namen smo uporabili postopek laserskega nanašanja vanadijevega karbidnega pragu (angl. Vanadium Carbide - VC ) na zlitino Ti-6Al-4V. Uporabili smo YAG vlakenski laser z močmi $1000 \mathrm{~W}, 1500 \mathrm{~W}$ in $2000 \mathrm{~W}$ ter hitrostjo nanašanja 4 $\mathrm{mm} / \mathrm{s}$. To so bili procesni parametri, ki smo jih uporabili za lasersko nanašanje vanadijevega karbidnega prahu na osnovni material (vzorec) iz Ti-6Al-4V zlitine, s čimer smo dosegli učvrstitev vrhnjega sloja vzorca. Debelina nanešenega sloja je naraščala $\mathrm{z}$ večanjem moči laserja. Nanešen sloj se je dobro spojil z osnovnim materialom, še posebej pri višjih procesnih močeh laserja. Pri močeh $2000 \mathrm{~W}$ in $1500 \mathrm{~W}$ se je vanadijev karbidni prah homogeno porazdelil po nanešenem sloju, medtem ko so se pri moči $1000 \mathrm{~W}$ pokazale nekatere neenakomerne porazdelitve prahu. Del prahu oziroma delcev vanadijevega karbida se je med laserskim nanašanjem najprej stalil in nato ponovno strdil v obliki dolgih dendritnih struktur. Nanešeni sloji so imeli bistveno višjo trdoto in odpornost proti obrabi (ugotovili smo skoraj dvakrat višje vrednosti), in to ne glede na procesne parametre nanašanja. Pri nižjih procesnih močeh, smo dobili višjo trdoto nanešenih slojev. Ugotovili smo tudi, da so sloji zelo dobro obstojni proti koroziji.
\end{abstract}

\section{PODATKI O ČLANKU}

Ključne besede: Lasersko nanašanje Zlitina Ti-6Al-4V

Vanadijev karbidni prah

Površinska mikrotrdota

Odpornost proti obrabi in koroziji

*Kontaktna oseba: emahoud@kku.edu.sa (Mahmoud, E.R.I.)

Zgodovina članka:

Prejet 2. avgusta 2014

Popravljen 29. oktobra 2014

Sprejet 10. novembra 2014 